\title{
RESPONSE DUE TO CONCENTRATED FORCE IN MICROPOLAR ELASTIC SOLID WITH VOIDS
}

\author{
R. SINGH* and K. SINGH \\ Department of Mathematics, S.G.A.D. Govt. College \\ Tarn Taran-143411, Punjab, INDIA \\ E-mail: kalsi_ranjit@yahoo.com \\ Star3003star@yahoo.co.in
}

\begin{abstract}
The eigen value approach, following Laplace and Fourier transforms has been employed to find the general solution of the field equation in a micropolar elastic solid with voids for the plane strain problem. An application of an infinite space with impulsive force has been taken to illustrate the utility of the approach. The integral transformations have been inverted by using a numerical inversion technique to get result in physical domain. The result in the form of normal displacement, volume fraction, normal force stress, tangential force stress and tangential couple stress components has been obtained numerically and illustrated graphically to depict the effect of micropolarity and voids.
\end{abstract}

Key words: eigen value, voids, Laplace and Fourier transform, concentrated force, Romberg's integration.

\section{Introduction}

The linear theory of elastic materials with voids is one of the generalizations of the classical theory of elasticity. This theory has practical utility to investigate various types of geological, biological and synthetic porous materials for which the elastic theory is inadequate.

The mechanical behavior of granular masses is strongly affected by their microstructure, namely the relative arrangement of voids and particles, i.e., the granular fabric. The study of deformations in granular materials is important in many areas of the science and technology, such as powder metallurgy and earthquake engineering. In recent years, dynamic compaction of powders has been used to manufacture advance composite. A granular medium is composed of a large number of distinct particles as well as some heterogeneous inclusions and voids filled with gas or liquid at the boundaries of discontinuity. Mismatch and incident waves will produce both transmission and reflection waves of different modes. Wave propagation phenomenon in such media not only depends on the microstructure but on the existence of inclusions and voids.

A non-linear theory concerning solid elastic materials consisting of various pores (voids) distributed throughout the body has been formulated by Nunziato and Cowin (1979). Lewis and Isaak (1982) discussed the voids of minimum stress concentration. Later, Cowin and Nunziato (1983) developed a theory of linear elastic materials with voids, for the mathematical study of the mechanical behavior of porous solids. Chandersekharahaiah (1989) discussed the problems of complete solution in the theory of isotropic elastic materials with voids. Some theorems of uniqueness for linear elastic materials with voids was proved by Scarpetta (1995). Cowin and Nunziato (1983) introduced the presence of pores in the classical continuum model by assigning an additional degree of freedom to each material particle. Consequently, the bulk mass density of such materials is given by the product of two fields, the void volume fraction and the mass density of matrix material. Classical mechanics deals with the basic assumption that the effect of the microstructure of a material is not essential for describing mechanical behavior. Such an approximation has been shown in

\footnotetext{
* To whom correspondence should be addressed
} 
many well-known cases. Often, however, discrepancies between the classical theory and experiments are observed, indicating that the microstructures might be important. For example, discrepancies have been found in the stress concentrations in the areas of holes, notches and cracks; elastic vibrations characterized by a high frequency and small wavelengths, particularly in granular composites consisting of stiff inclusions embedded in a weaker matrix, fibers or grains; and the mechanical behavior of complex fluids such as liquid crystals, polymeric suspensions, and animal blood.

Various degrees of freedom of a microstructure were considered by different authors, e.g., Cosserat and Cosserat (1909), Eringen and Suhubi (1964) and Mindlin (1964). The force at a point of a surface element of the body of these materials is completely characterized by a stress vector and a couple stress vector at that point. In the classical theory of elasticity, the effect of couple stress is neglected. Eringen (1966) modified his earlier theory and renamed it as the "Linear Theory of Micropolar Elasticity".

Scarpetta (1990) worked on the fundamental solution in micropolar elasticity with voids. Marin (1996a) obtains the existence and uniqueness of solutions for boundary value problems in elasticity of micropolar materials with voids. Marin (1996b) discussed generalized solutions in elasticity of micropolar bodies with voids. Marin (1998) derived an evolutionary equation for a micropolar elastic body with voids. Kumar et al. (2002), discussed the surface wave propagation in a micropolar liquid saturated porous layer. Kumar and Ailawalia (2007) studied the interaction in a micropolar thermoelastic medium with voids due to distributed loads. Kumar and Ailawalia (2009) studied the influence of various sources in a micropolar thermoelastic medium with voids. Madeo and Gavrilyuk (2010) studied the propagation of acoustic waves in porous media and their reflection and transmission at a purefluid/ porous-medium permeable interface. Tomar and Khurana (2011) investigated the reflection and transmission phenomena of a plane longitudinal wave from a plane interface between two distinct micropolar porous elastic solid half spaces in welded contact. Kumar and Kumar (2011) studied the wave propagation in an orthotropic generalized thermoelastic half space with voids under initial stress. Singh and Singh (2013) investigated eigen value approach in a micropolar elastic medium with voids.

\section{Formulation and solution of the problem}

We consider a homogeneous, isotropic micropolar elastic solid with voids. We take a Cartesian coordinate system $(x, y, z)$ and the $z$-axis is pointed into the medium. Following Eringen (1968) and Iesan (1985) the field equations and the constitutive relations in a micropolar elastic solid with voids in the absence of body forces are given by as

$$
\begin{aligned}
& \beta^{*} \nabla q+(\lambda+2 \mu+K) \nabla(\nabla \cdot \boldsymbol{u})-(\mu+K) \times \nabla \times \nabla \times \boldsymbol{u}+K \nabla \times \varphi=\rho \frac{\partial^{2} \boldsymbol{u}}{\partial t^{2}}, \\
& (\alpha+\beta+\gamma) \nabla(\nabla \cdot \varphi)-\gamma \nabla \times \nabla \times \varphi+K \nabla \times \varphi-2 K \boldsymbol{\varphi}=\rho j \frac{\partial^{2} \boldsymbol{\varphi}}{\partial t^{2}}, \\
& \alpha^{*} \nabla^{2} q^{*}-\zeta^{*} q^{*}-\omega^{*} \frac{\partial q^{*}}{\partial t}-\beta^{*} \nabla \cdot \boldsymbol{u}=\rho K^{*} \frac{\partial^{2} q^{*}}{\partial t^{2}}, \\
& t_{i j}=\left(\beta^{*} q^{*}+\lambda u_{r r}\right) \delta_{i j}+\mu\left(u_{i, j}+u_{j, i}\right)+K\left(u_{i, j}-\varepsilon_{i j r} \varphi_{r}\right), \\
& m_{i j}=\alpha \varphi_{r r} \delta_{j i}+\beta \varphi_{j, i}+\gamma \varphi_{j, i} .
\end{aligned}
$$

Since we are considering a two-dimensional axisymmetric problem, so we assume the components of the displacement vector $\boldsymbol{u}$ and microrotation vector $\varphi$ of the form 


$$
\boldsymbol{u}=\left(u_{1}, 0, u_{3}\right), \quad \boldsymbol{\varphi}=\left(0, \varphi_{2}, 0\right) .
$$

Due to the symmetry about the $z$-axis all the quantities are independent of $y$. Using Eqs (2.6) the set of Eqs (2.1) - (2.3) reduces to

$$
\begin{aligned}
& \beta^{*} \frac{\partial q^{*}}{\partial x}+(\lambda+\mu)\left(\frac{\partial^{2} u_{1}}{\partial x^{2}}+\frac{\partial^{2} u_{3}}{\partial x \partial z}\right)-K \frac{\partial \varphi_{2}}{\partial z}+(\mu+K) \nabla^{2} u_{1}=\rho \frac{\partial^{2} u_{1}}{\partial t^{2}} \\
& \beta^{*} \frac{\partial q^{*}}{\partial x}+(\lambda+\mu)\left(\frac{\partial^{2} u_{3}}{\partial z^{2}}+\frac{\partial^{2} u_{1}}{\partial x \partial z}\right)-K \frac{\partial \varphi_{2}}{\partial z}+(\mu+K) \nabla^{2} u_{3}+K \frac{\partial \varphi_{2}}{\partial x}=\rho \frac{\partial^{2} u_{3}}{\partial t^{2}} \\
& \gamma \nabla^{2} \varphi_{2}+K\left(\frac{\partial u_{1}}{\partial z}-\frac{\partial u_{3}}{\partial x}\right)-2 K \varphi_{2}=\rho j \frac{\partial^{2} \varphi_{2}}{\partial t^{2}}, \\
& \alpha^{*} \nabla^{2} q^{*}-\zeta^{*} q^{*}-\omega^{*} \frac{\partial q^{*}}{\partial t}-\beta^{*}\left(\frac{\partial u_{1}}{\partial x}+\frac{\partial u_{3}}{\partial z}\right)=\rho K^{*} \frac{\partial^{2} q^{*}}{\partial t^{2}},
\end{aligned}
$$

where

$$
\nabla^{2}=\frac{\partial^{2}}{\partial x^{2}}+\frac{\partial^{2}}{\partial z^{2}}
$$

Using Eqs (2.6) and introducing dimensionless quantities as

$$
\begin{aligned}
& x^{\prime}=\frac{\omega_{1}}{c_{1}} x, \quad z^{\prime}=\frac{\omega_{1}}{c_{1}} z, \quad u_{1}^{\prime}=\frac{\omega_{1}}{c_{1}} u_{1}, \quad u_{3}^{\prime}=\frac{\omega_{1}}{c_{1}} u_{3}, \quad \varphi_{2}^{\prime}=\frac{\omega_{1}^{2}}{c_{1}^{2}} \varphi_{2}, \quad t^{\prime}=\omega_{1} t, \\
& q^{* \prime}=\frac{\omega_{1}^{2}}{c_{1}^{2}} q^{*}, \quad t_{33}^{\prime}=\frac{t_{33}}{\mu}, \quad t_{31}^{\prime}=\frac{t_{31}}{\mu}, \quad m_{32}^{\prime}=\frac{\omega_{1}}{c_{1}} m_{32},
\end{aligned}
$$

where

$$
c_{1}^{2}=\frac{(\lambda+2 \mu+K)}{\rho}, \quad \omega_{l}^{2}=\frac{K}{\rho j} .
$$

The set of Eqs (2.7) - (2.10) reduces to (after suppressing the primes for convenience)

$$
s_{0} \frac{\partial q^{*}}{\partial x}+\left(\frac{\partial^{2} u_{1}}{\partial x^{2}}+\frac{\partial^{2} u_{1}}{\partial x \partial z}\right)-s_{1} \nabla^{2} u_{1}-s_{2} \frac{\partial \varphi_{2}}{\partial z}=s_{3} \frac{\partial^{2} u_{1}}{\partial t^{2}}
$$




$$
\begin{aligned}
& s_{0} \frac{\partial q^{*}}{\partial z}+\left(\frac{\partial^{2} u_{1}}{\partial z^{2}}+\frac{\partial^{2} u_{1}}{\partial x \partial z}\right)-s_{1} \nabla^{2} u_{3}-s_{2} \frac{\partial \varphi_{2}}{\partial x}=s_{3} \frac{\partial^{2} u_{3}}{\partial t^{2}}, \\
& \left(\frac{\partial^{2} \varphi_{2}}{\partial x^{2}}+\frac{\partial^{2} \varphi_{2}}{\partial x \partial z}\right)-s_{4}\left(\frac{\partial u_{1}}{\partial z}-\frac{\partial u_{3}}{\partial x}\right)-s_{5} \varphi_{2}=s_{6} \frac{\partial^{2} \varphi_{2}}{\partial t^{2}}, \\
& \nabla^{2} q^{*}-s_{7} q^{*}-s_{8} \frac{\partial q^{*}}{\partial t}-s_{9}\left(\frac{\partial u_{3}}{\partial z}+\frac{\partial u_{1}}{\partial x}\right)-s_{5} \varphi_{2}=s_{10} \frac{\partial^{2} q^{*}}{\partial t^{2}},
\end{aligned}
$$

where

$$
\begin{aligned}
& s_{0}=\frac{\beta^{*} c_{1}^{2}}{(\lambda+\mu) j \omega_{1}^{2}}, \quad s_{1}=\frac{(K+\mu)}{(\lambda+\mu)}, \quad s_{2}=\frac{K c_{1}^{2}}{(\lambda+\mu) j \omega_{1}^{2}}, \quad s_{3}=\frac{\rho c_{1}^{2}}{(\lambda+\mu)}, \\
& s_{4}=\frac{K j}{\gamma}, \quad s_{5}=\frac{2 K c_{1}^{2}}{\gamma \omega_{1}^{2}}, \quad s_{7}=\frac{\zeta^{*} c_{1}^{2}}{\alpha^{*} \omega_{1}^{2}}, \quad s_{8}=\frac{\omega^{*} c_{1}^{2}}{\alpha^{*} \omega_{1}}, \\
& s_{9}=\frac{\beta^{*} j}{\alpha^{*}}, \quad s_{10}=\frac{K^{*} \rho c_{1}^{2}}{\alpha^{*}},
\end{aligned}
$$

Now applying the Laplace and Fourier transforms defined by

$$
\bar{f}(x, z, p)=L\{f(x, z, t)\}=\int_{0}^{\infty} f(x, z, t) \exp (-p t) d t,
$$

and

$$
\tilde{f}(\xi, z, p)=F\{\bar{f}(x, z, p)\}=\int_{-\infty}^{\infty} \bar{f}(x, z, p) \exp (-1 \xi x) d x
$$

on the set of Eqs (2.13) - (2.16), we obtain,

$$
\begin{aligned}
& \frac{d^{2} \tilde{u}_{1}}{d z^{2}}=\frac{\left(\xi^{2}+s_{1} \xi^{2}+s_{1} p^{2}\right)}{s_{1}} \tilde{u}_{1}-\frac{1 \xi}{s_{1}} \frac{d \tilde{u}_{3}}{d z}+\frac{s_{2}}{s_{1}} \frac{d \tilde{\varphi}_{2}}{d z}+\frac{1 \xi s_{0}}{s_{1}} \tilde{q}^{*}, \\
& \frac{d^{2} \tilde{u}_{3}}{d z^{2}}=\frac{\left(s_{1} \xi^{2}+s_{3} p^{2}\right)}{1+s_{1}} \tilde{u}_{3}-\frac{1 \xi}{1+s_{1}} \frac{d \tilde{u}_{1}}{d z}-\frac{1 s_{0}}{1+s_{1}} \frac{d \tilde{\varphi}_{2}}{d z}-\frac{\xi s_{2}}{s_{1}} \tilde{q}^{*}, \\
& \frac{d^{2} \tilde{\varphi}_{2}}{d z^{2}}=-s_{4} \frac{d \tilde{u}_{3}}{d z}-1 \xi_{s_{4}} \tilde{u}_{1}+\left(\xi^{2}+s_{5}+s_{6} p^{2}\right) \tilde{\varphi}_{2},
\end{aligned}
$$




$$
\frac{d^{2} \tilde{q}^{*}}{d z^{2}}=-s_{9} \frac{d \tilde{u}_{1}}{d z}-1 \xi_{s_{9}} \tilde{u}_{1}+\left(\xi^{2}+s_{7}+s_{8} p+s_{10} p^{2}\right) \tilde{q}^{*},
$$

The system of Eqs (2.20) - (2.23) can be written in the vector matrix differential equation form as

$$
\frac{d W(\xi, z, p)}{d z}=A(\xi, p) W(\xi, z, p)
$$

where

$$
\begin{aligned}
& W=\left[\begin{array}{c}
U \\
D U
\end{array}\right], \quad A=\left[\begin{array}{ll}
0 & I \\
A_{2} & A_{1}
\end{array}\right], \quad U=\left[\begin{array}{l}
\tilde{u}_{1} \\
\tilde{\varphi}_{2} \\
\tilde{q}^{*}
\end{array}\right], \\
& A_{1}=\left[\begin{array}{cccc}
0 & a_{12} & a_{13} & 0 \\
a_{21} & 0 & 0 & a_{24} \\
a_{31} & 0 & 0 & 0 \\
0 & a_{24} & 0 & 0
\end{array}\right], \quad A_{2}=\left[\begin{array}{cccc}
b_{11} & 0 & 0 & b_{14} \\
0 & b_{22} & b_{23} & 0 \\
0 & b_{32} & b_{33} & 0 \\
b_{41} & 0 & 0 & b_{44}
\end{array}\right], \\
& a_{12}=\frac{1 \xi}{s_{1}}, \quad a_{13}=\frac{s_{2}}{s_{1}}, \quad a_{21}=-\frac{1 \xi}{1+s_{1}}, \quad \begin{array}{c}
a_{24}=\frac{-s_{0}}{1+s_{1}}, \quad D=\frac{d}{d z}, \\
a_{31}=-s_{4}, \quad a_{42}=s_{9},
\end{array} b_{11}=\frac{\left(\xi^{2}+s_{1} \xi^{2}+s_{1} p^{2}\right)}{s_{1}}, \quad b_{14}=-\frac{1 s_{0} \xi^{2}}{s_{1}}, \\
& b_{22}=\frac{\left(s_{1} \xi^{2}+s_{3} p^{2}\right)}{1+s_{1}}, \quad b_{23}=\frac{-1 s_{2} \xi^{2}}{1+s_{1}}, \quad b_{32}=1 s_{4} \xi^{2}, \\
& b_{33}=\left(\xi^{2}+s_{5}+s_{6} p^{2}\right), \quad b_{44}=\left(\xi^{2}+s_{7}+s_{8} p+s_{10} p^{2}\right),
\end{aligned}
$$

and $\mathrm{O}$ is the null matrix and $\mathrm{I}$ is the unit matrix of order 4

To solve Eq.(2.24), we take

$$
W(\xi, z, p)=W(\xi, p) e^{q z}
$$

For some parameter $q$, so we obtain

$$
A(\xi, p) W(\xi, z, p)=q W(\xi, z, p)
$$

which leads to an eigen value problem. The characteristic equation corresponding to the matrix $\mathrm{A}$ is given by

$$
\operatorname{det}(A-q I)=0
$$


which on expansion provides us

$$
q^{8}-\sigma_{1} q^{6}+\sigma_{2} q^{4}-\sigma_{3} q^{2}+\sigma_{4}=0
$$

where

$$
\begin{aligned}
\sigma_{1}= & b_{11}+b_{22}+b_{33}+b_{44}+a_{24} a_{42}+a_{12} a_{21}+a_{13} a_{31}, \\
\sigma_{2}= & b_{11} b_{22}+b_{22} b_{33}+b_{11} b_{33}+b_{11} b_{44}+b_{22} b_{44}+b_{33} b_{44}-b_{41} a_{24} a_{12}+ \\
& +b_{11} a_{24} a_{42}+b_{33} a_{24} a_{42}+a_{24} a_{42} a_{13} a_{31}-b_{32} b_{23}+a_{12} a_{21} b_{33}+a_{12} a_{21} b_{44}+ \\
& -a_{12} a_{31} b_{23}-a_{31} a_{21} b_{32}+a_{13} a_{31} b_{44}-b_{14} b_{41}-b_{14} a_{21} a_{42}, \\
\sigma_{3}= & b_{11} b_{22} b_{33}+b_{22} b_{33} b_{44}+b_{11} b_{33} b_{44}+b_{11} b_{22} b_{44}-b_{11} b_{23} b_{32}-b_{44} b_{23} b_{32}+ \\
& a_{42} a_{42} b_{11} b_{33}+a_{12} a_{24} b_{41} b_{32}+a_{31} a_{42} b_{41} b_{23}-a_{31} a_{42} b_{14} b_{23}+a_{12} a_{21} b_{33} b_{44}+ \\
& -a_{13} a_{21} b_{32} b_{45}-a_{12} a_{31} b_{23} b_{44}-a_{12} a_{24} b_{41} b_{33}-a_{42} a_{21} b_{14} b_{33}+a_{13} a_{31} b_{22} b_{44}+ \\
& -b_{14} b_{41} b_{22}-b_{14} b_{41} b_{33}, \\
\sigma_{4}= & b_{11} b_{22} b_{33} b_{44}-b_{11} b_{44} b_{23} b_{32}-b_{14} b_{41} b_{22} b_{33}+b_{14} b_{41} b_{23} b_{32},
\end{aligned}
$$

The eigen values of the matrix A are characteristic roots of the (2.29). The eigen vectors $X(\xi, p)$ corresponding to the eigen value $q_{s}$ can be determined by solving the homogenous equations

$$
[A-q I X(\xi, p)=0
$$

The set of eigen vectors $X_{s}(\xi, p) ; s=1,2,3, \ldots \ldots .8$ may be defined as

$$
X_{s}(\xi, p)=\left[\begin{array}{c}
X_{s 1}(\xi, p) \\
X_{s 2}(\xi, p)
\end{array}\right]
$$

where

$$
\begin{aligned}
X_{s 1}=\left[\begin{array}{c}
a_{s} q_{s} \\
b_{s} \\
-\xi \\
c_{s}
\end{array}\right], & X_{s 1}=\left[\begin{array}{c}
a_{s} q_{s}^{2} \\
b_{s} q_{s} \\
-\xi q_{s} \\
c_{s} q_{s}
\end{array}\right], \quad q=q_{s} ; \quad s=1,2,3,4 \\
X_{l 1}=\left[\begin{array}{c}
a_{s} q_{s} \\
b_{s} \\
-\xi \\
c_{s}
\end{array}\right], & X_{l 2}=\left[\begin{array}{c}
a_{s} q_{s}^{2} \\
b_{s} q_{s} \\
-\xi q_{s} \\
c_{s} q_{s}
\end{array}\right], \quad l=s+4, \quad q=-q_{s} ; \quad s=1,2,3,4
\end{aligned}
$$




$$
\begin{aligned}
a_{s}= & \frac{\xi}{\Delta_{s}}\left\{\left(\xi^{2}+s_{7}+s_{8} p+s_{10} p^{2}\right)\left(\xi^{2}+s_{5}+s_{6} p^{2}+s_{2} s_{4}-q_{s}^{2}\right)-s_{2} s_{4} q_{s}^{2}+\right. \\
& \left.-\left(\xi^{2}+s_{5}+s_{6} p^{2}-q_{s}^{2}\right)\left(s_{0} s_{9}-q_{s}^{2}\right)\right\}, \\
b_{s}= & \frac{-1}{\Delta_{s}}\left\{( \xi ^ { 2 } + s _ { 7 } + s _ { 8 } p + s _ { 1 0 } p ^ { 2 } - q _ { s } ^ { 2 } ) \left\{\left(\xi^{2}+s_{1} \xi^{2}+s_{3} p^{2}-s_{1} q_{s}^{2}\right)\left(\xi^{2}+s_{5}+s_{6} p^{2}-q_{s}^{2}\right)\right.\right. \\
& \left.\left.+s_{2} s_{4} q_{s}^{2}\right\}-s_{0} s_{9} \xi^{2}\left(\xi^{2}+s_{5}+s_{6} p^{2}-q_{s}^{2}\right)\right\}, \\
c_{s}= & \frac{s_{9}\left(1 \xi_{9}+b_{s}\right)}{\left\{q_{s}^{2}-\left(\xi^{2}+s_{7}+s_{8} p+s_{10} p^{2}\right)\right\}}, \\
\Delta_{s}= & s_{4}\left[s_{0} s_{9}\left(q_{s}^{2}-\xi^{2}\right)-\left(\xi^{2}+s_{7}+s_{8} p+s_{10} p^{2}-q_{s}^{2}\right)\left\{q_{s}^{2}-\left(\xi^{2}+s_{1} \xi^{2}+s_{3} p^{2}-s_{1} q_{s}^{2}\right)\right\}\right], \\
s_{=} & 1,2,3,4
\end{aligned}
$$

Thus solution of Eq.(2.24) is as given by Sharma and Chand (1992)

$$
W(\xi, z, p)=\sum_{s=1}^{4}\left[E_{S} X_{s} \exp \left(q_{s} z\right)+E_{s+4} X_{s+4} \exp \left(-q_{s} z\right)\right]
$$

where $E_{1}, E_{2}, E_{3}, E_{4}, E_{5}, E_{6}, E_{7}$ and $E_{8}$ are eight arbitrary constants. Equation (2.42) represents a general solution of the plane strain problem for an isotropic, micropolar elastic solid with voids and gives displacement, microrotation and volume fraction components in the transformed domain.

\section{Application}

\section{Case-I Mechanical Normal Point Source}

We consider an infinite micropolar elastic space with voids in which a concentrated force $F=-F_{0} \delta(x) \delta(t)$ where $F_{0}$ is the magnitude of the force, acting in the direction of the $z$-axis at the origin of the Cartesian co-ordinate system as shown in Fig.1. The boundary conditions for the present problem on the plane $\boldsymbol{z}=0$ are 


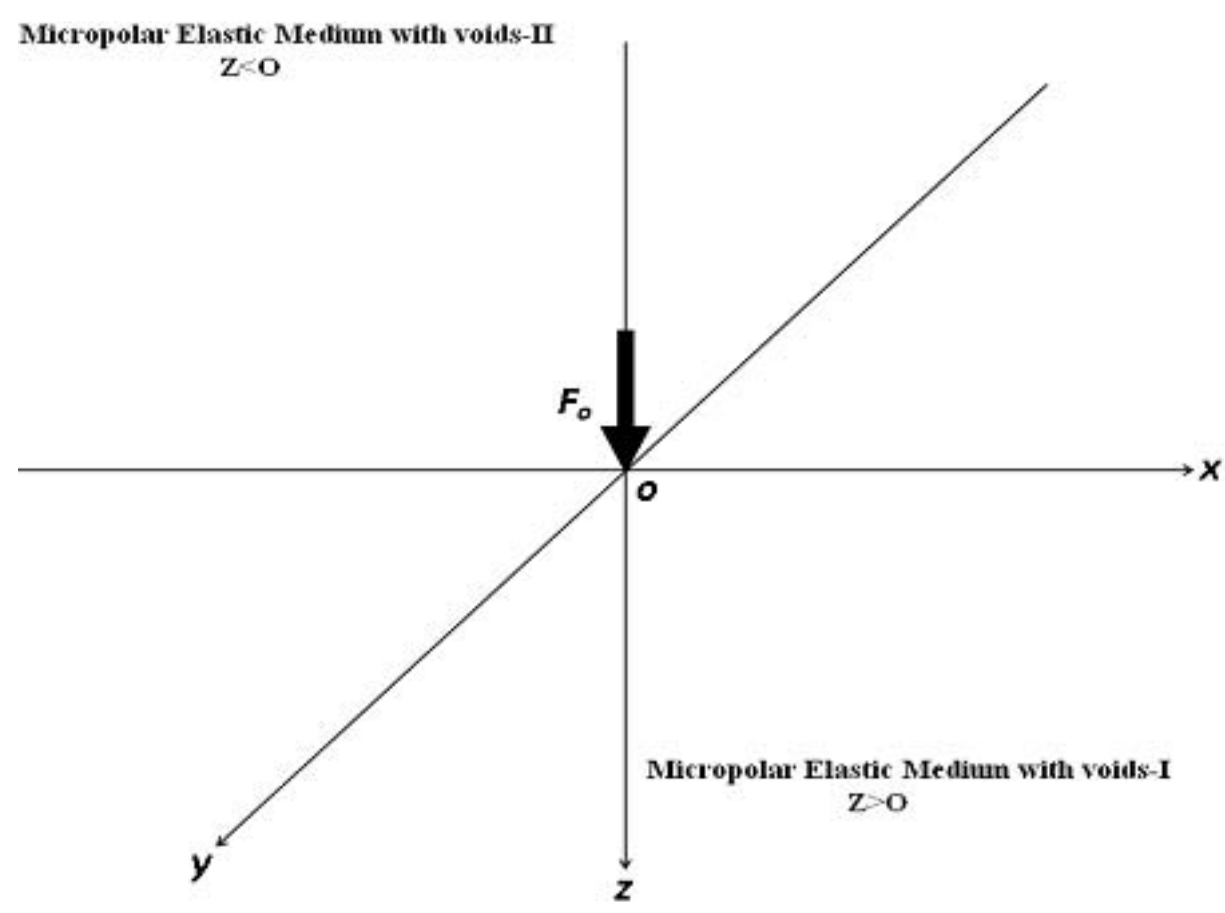

Fig.1. Geometry of the problem.

$$
\begin{array}{ll}
u_{1}\left(x, 0^{+}, t\right)-u_{1}\left(x, 0^{-}, t\right)=0, & u_{3}\left(x, 0^{+}, t\right)-u_{3}\left(x, 0^{-}, t\right)=0, \\
\varphi_{2}\left(x, 0^{+}, t\right)-\varphi_{2}\left(x, 0^{-}, t\right)=0, & q^{*}\left(x, 0^{+}, t\right)-q^{*}\left(x, 0^{-}, t\right)=0, \\
t_{31}\left(x, 0^{+}, t\right)-t_{31}\left(x, 0^{-}, t\right)=0, & t_{33}\left(x, 0^{+}, t\right)-t_{33}\left(x, 0^{-}, t\right)=-F_{0} \delta(x) \delta(t), \\
m_{32}\left(x, 0^{+}, t\right)-m_{32}\left(x, 0^{-}, t\right)=0, & \frac{\partial q^{*}\left(x, 0^{+}, t\right)}{\partial z}-\frac{\partial q^{*}\left(x, 0^{-}, t\right)}{\partial z}=0 .
\end{array}
$$

Making use of Eqs (2.6), (2.12) and $F_{0}^{\prime}=\frac{F_{0}}{K}$ on Eqs (2.4) - (2.5) and we get the stresses in the nondimensional form with primes. After suppressing the primes, we apply the Laplace and Fourier transforms defined by Eqs (2.18) and (2.19) on the resulting equations and from Eqs (3.1)-(3.4), we get the transformed components of displacement, microrotation, volume fraction, tangential force stress, normal force stress and tangential couple stress for $z>0$ which are given by

$$
\begin{aligned}
& \tilde{u}_{1}(\xi, z, p)=\left\{a_{1} q_{1} E_{5} e^{-q_{1} z}+a_{2} q_{2} E_{6} e^{-q_{2} z}+a_{3} q_{3} E_{7} e^{-q_{3} z}+a_{4} q_{4} E_{8} e^{-q_{4} z}\right\}, \\
& \tilde{u}_{3}(\xi, z, p)=b_{1} E_{5} e^{-q_{1} z}+b_{2} E_{6} e^{-q_{2} z}+b_{3} E_{7} e^{-q_{3} z}+b_{4} E_{8} e^{-q_{4} z}, \\
& \tilde{\varphi}_{2}(\xi, z, p)=-\xi\left\{E_{5} e^{-q_{1} z}+E_{6} e^{-q_{2} z}+E_{7} e^{-q_{3} z}+E_{8} e^{-q_{4} z}\right\},
\end{aligned}
$$




$$
\begin{aligned}
& \tilde{q}^{*}(\xi, z, p)=c_{1} E_{5} e^{-q_{1} z}+c_{2} E_{6} e^{-q_{2} z}+c_{3} E_{7} e^{-q_{3} z}+c_{4} E_{8} e^{-q_{4} z} \\
& \tilde{t}_{31}(\xi, z, p)=\left(s_{11} a_{1} q_{1}^{2}+\xi b_{1}+\xi s_{12}\right) E_{5} e^{-q_{1} z}+\left(s_{11} a_{2} q_{2}^{2}-\xi b_{2}+\xi s_{12}\right) E_{6} e^{-q_{2} z}+ \\
& +\left(s_{11} a_{3} q_{3}^{2}-\xi b_{3}+\xi s_{12}\right) E_{7} e^{-q_{3} z}+\left(s_{11} a_{4} q_{4}^{2}-\xi b_{4}+\xi s_{12}\right) E_{8} e^{-q_{4} z} \\
& \tilde{t}_{33}(\xi, z, p)=\left[\left(s_{13} c_{1}-s_{14} \xi a_{1} q_{1}+s_{15} b_{1} q_{1}\right) E_{5} e^{-q_{1} z}+\right. \\
& +\left(s_{13} c_{2}-s_{14} \xi a_{2} q_{2}+s_{15} b_{2} q_{2}\right) E_{6} e^{-q_{2} z}+\left(s_{13} c_{3}-s_{14} \xi a_{3} q_{3}+s_{15} b_{3} q_{3}\right) E_{7} e^{-q_{3} z}+ \\
& \left.+\left(s_{13} c_{4}-s_{14} \xi a_{4} q_{4}+s_{15} b_{4} q_{4}\right) E_{8} e^{-q_{4} z}\right] \\
& \tilde{m}_{32}(\xi, z, p)=\xi\left\{q_{1} E_{1} e^{-q_{1} z}+q_{2} E_{6} e^{-q_{2} z}+q_{3} E_{7} e^{-q_{3} z}+q_{4} E_{8} e^{-q_{4} z}\right\}
\end{aligned}
$$

for $z<0$, the above expressions get suitably modified, e.g.,

$$
\tilde{u}_{1}(\xi, z, p)=\left\{a_{1} q_{1} E_{1} e^{-q_{1} z}+a_{2} q_{2} E_{2} e^{-q_{2} z}+a_{3} q_{3} E_{3} e^{-q_{3} z}+a_{4} q_{4} E_{4} e^{-q_{4} z}\right\}
$$

Making use of the transformed displacements, microrotation, volume fraction and stress components given by Eqs (3.5)-(3.12) in the boundary conditions, we obtain eight linear relations between $E_{1}, E_{2}, E_{3}, E_{4}, E_{5}, E_{6}, E_{7}$ and $E_{8}$, which on solving give

$$
\begin{aligned}
& E_{1}=E_{5}=\frac{F_{0}}{4 \pi q_{1} \Delta_{l}^{*}}\left[c_{2}\left(a_{3}-a_{4}\right)+c_{3}\left(a_{4}-a_{2}\right)+c_{4}\left(a_{2}-a_{3}\right)\right], \\
& E_{2}=E_{6}=\frac{F_{0}}{4 \pi q_{2} \Delta_{l}^{*}}\left[c_{1}\left(a_{4}-a_{3}\right)+c_{3}\left(a_{1}-a_{4}\right)+c_{4}\left(a_{3}-a_{1}\right)\right], \\
& E_{3}=E_{7}=\frac{F_{0}}{4 \pi q_{3} \Delta_{l}^{*}}\left[c_{1}\left(a_{2}-a_{4}\right)+c_{2}\left(a_{4}-a_{1}\right)+c_{4}\left(a_{1}-a_{2}\right)\right], \\
& E_{4}=E_{8}=\frac{F_{0}}{4 \pi q_{4} \Delta_{l}^{*}}\left[c_{1}\left(a_{3}-a_{2}\right)+c_{2}\left(a_{1}-a_{3}\right)+c_{3}\left(a_{2}-a_{1}\right)\right],
\end{aligned}
$$

where

$$
\begin{aligned}
\Delta_{1}^{*}=s_{15} & {\left[c_{1}\left\{\left(a_{2} b_{3}-a_{3} b_{2}\right)+\left(a_{3} b_{4}-a_{4} b_{3}\right)+\left(a_{4} b_{2}-a_{2} b_{4}\right)\right\}+\right.} \\
& +c_{2}\left\{\left(a_{3} b_{1}-a_{1} b_{3}\right)+\left(a_{1} b_{4}-a_{4} b_{1}\right)+\left(a_{4} b_{3}-a_{3} b_{4}\right)\right\}+ \\
& +c_{3}\left\{\left(a_{1} b_{2}-a_{2} b_{1}\right)+\left(a_{4} b_{1}-a_{1} b_{4}\right)+\left(a_{2} b_{4}-a_{4} b_{2}\right)\right\}+ \\
& \left.+c_{4}\left\{\left(a_{2} b_{1}-a_{1} b_{2}\right)+\left(a_{1} b_{3}-a_{3} b_{1}\right)+\left(a_{3} b_{2}-a_{2} b_{3}\right)\right\}\right] .
\end{aligned}
$$

Thus functions $\tilde{u}_{1}, \tilde{u}_{3}, \tilde{\varphi}_{2}, \tilde{T}, \tilde{t}_{31}, \tilde{t}_{33}$ and $\tilde{m}_{32}$ have been determined in the transform domain and they enable us to find the displacements, microrotation, temperature field and stresses. 


\section{Case-II Mechanical Continuous Normal Point Source}

When the plane boundary is subjected to continuous normal point force, the boundary conditions at the interface $z=0$ are

$$
\begin{array}{ll}
u_{1}\left(x, 0^{+}, t\right)-u_{1}\left(x, 0^{-}, t\right)=0, & u_{3}\left(x, 0^{+}, t\right)-u_{3}\left(x, 0^{-}, t\right)=0, \\
\varphi_{2}\left(x, 0^{+}, t\right)-\varphi_{2}\left(x, 0^{-}, t\right)=0, & q^{*}\left(x, 0^{+}, t\right)-q^{*}\left(x, 0^{-}, t\right)=0, \\
t_{32}\left(x, 0^{+}, t\right)-t_{32}\left(x, 0^{-}, t\right)=0, & t_{33}\left(x, 0^{+}, t\right)-t_{33}\left(x, 0^{-}, t\right)=-F_{0} \delta(x) H(t), \\
m_{32}\left(x, 0^{+}, t\right)-m_{32}\left(x, 0^{-}, t\right)=0, & \frac{\partial q^{*}\left(x, 0^{+}, t\right)}{\partial z}-\frac{\partial q^{*}\left(x, 0^{-}, t\right)}{\partial z}=0,
\end{array}
$$

where $F_{0}$ is the magnitude of the force applied and $\mathrm{H}(\mathrm{t})$ is Heaviside distribution. With the help of these boundary conditions (3.18) - (3.21), the expressions for the components of displacement, force stress, couple stress and volume fraction field are obtained by Eqs (3.5)-(3.12) replacing $\Delta_{l}^{*}$ with $\Delta_{l}^{*} / p$.

Particular Case I: Neglecting the influence of voids, i.e., $\left(\alpha^{*}=\beta^{*}=\zeta^{*}=\omega^{*}=K^{*}=0\right)$; the expressions for the displacement components, force stresses and couple stress are obtained in a micropolar elastic medium.

Particular Case II: If the effect of micropolarity is ignored, i.e., $(K=j=\alpha=\beta=\gamma=0)$; the expressions for the displacement components, force stresses and volume fraction field are obtained in an elastic medium with voids.

Particular Case III: If the effect of micropolarity and voids is neglected, i.e., $(K=j=\alpha=\beta=\gamma=0)$ and $\left(\alpha^{*}=\beta^{*}=\zeta^{*}=\omega^{*}=K^{*}=0\right)$; the expressions for the displacement components and force stresses are obtained in an elastic medium.

\section{Method for the inversion of transforms}

The transformed solutions are the functions of the form $\tilde{f}(\xi, z, p)$, and to get the function $f(r, z, p)$, first we invert the Fourier transform by using

$$
\tilde{f}(\xi, z, p)=\int_{-\infty}^{\infty} \bar{f}(x, z, p) e(-1 \xi x) d x
$$

The expression (4.1) gives us the Laplace transform $\bar{f}(\xi, z, p)$ of the function $\bar{f}(\xi, z, p)$. Now, for the fixed values of $r$ and $z$ the function $\bar{f}(\xi, z, p)$ can be considered as the Laplace transform $\bar{g}(p)$ of some function $g(t)$. Following Honig and Hirdes (1984), the Laplace transformed function $\bar{g}(p)$ can be inverted numerically as given below.

The function $g(t)$ can be obtained from $\bar{g}(p)$ by using the inversion formula 


$$
g(t)=\frac{1}{2 \imath \pi} \int_{c+\infty}^{c+\infty} e^{p t} \bar{g}(p) d \xi
$$

where $C$ is an arbitrary real number greater than all the real parts of the singularities of $\bar{g}(p)$. The actual procedure to invert the Laplace transform consists of Eq.(4.2) together with the $\varepsilon$-algorithm. The values of $C$ and $L$ are chosen according to the criteria outlined by Honig and Hirdes (1984).

The last step is to calculate the integral in Eq.(4.1).The method for evaluating this integral is described by Press et al. (1986), which involves the use of Romberg's integration with adaptive step size. It also uses the results from successive refinements of the extended trapezoidal rule followed by extrapolation of the results to the limit when the step size tends to zero.

\section{Numerical results and discussion} crystal

Following Eringen (1984), we take the following values of relevant parameters for the magnesium

$$
\begin{aligned}
& \lambda=9.4 \times 10^{10} \mathrm{~N} / \mathrm{m}^{2}, \quad \mu=4 \times 10^{10} \mathrm{~N} / \mathrm{m}^{2}, \quad \rho=1.74 \times 10^{3} \mathrm{~kg} / \mathrm{m}^{3}, \quad \gamma=0.779 \times 10^{-9} \mathrm{~N}, \\
& j=0.2 \times 10^{-19} \mathrm{~m}^{2}, \quad \alpha^{\#}=3.688 \times 10^{-9} \mathrm{~N}, \quad \beta^{\#}=1.138494 \times 10^{10} \mathrm{~N} / \mathrm{m}^{2}, \\
& \zeta^{\#}=1.1475 \times 10^{10} \mathrm{~N} / \mathrm{m}^{2}, \quad \omega^{\#}=0.0787 \times 10^{-1} \mathrm{~N} \times \mathrm{sec} / \mathrm{m}^{2}, \quad K^{\#}=1.1753 \times 10^{-19} \mathrm{~m}^{2} .
\end{aligned}
$$

The computations were carried out for non-dimensional time $t=0.1$ at $z=1$ in the range $0 \leq x \leq 8$. The variations of non-dimensional normal displacement, $U_{3}\left(=2 u_{3} / F_{0}\right)$ non-dimensional volume fraction, $Q\left(=2 q^{*} / F_{0}\right)$ non-dimensional normal stress and $T_{33}\left(=2 t_{33} / F_{0}\right)$ non-dimensional tangential couple stress with $M_{32}\left(=2 m_{32} / F_{0}\right)$ non-dimensional distance ' $x$ ' are shown in Figs 2-5. The solid line gives the variations of components for a micropolar elastic solid with voids (MESV) whereas the very small dashed lines are for a micropolar elastic solid (MES), these lines correspond to the variations for a micropolar elastic solid with voids (ESV) and large dashed lines are for an elastic solid (ES).

\section{Mechanical normal point source}

The variations of normal displacement, volume fraction field, normal force stress and tangential couple stress with distance ' $x$ ' for MESV, MES, EVS and ES when a mechanical normal point source is applied are shown in Figs 2, 3, 4,5, respectively.

The variations of normal displacement $U_{3}$ with $x$ for all four theories (MESV,MES, ESV, ES) are shown in Fig.2 and it is observed that the behavior of $U_{3}$ for MES is opposite to MESV, ESV and ES. The values of $U_{3}$ decrease sharply as $r$ lies between $0 \leq x \leq 3$ whereas for MES the values of $U_{3}$ increase in the same range. Very near to the point of action of sources, the magnitude of values of $U_{3}$ is larger for MESV and smallest for MES, as the source is very near to the point of action. 


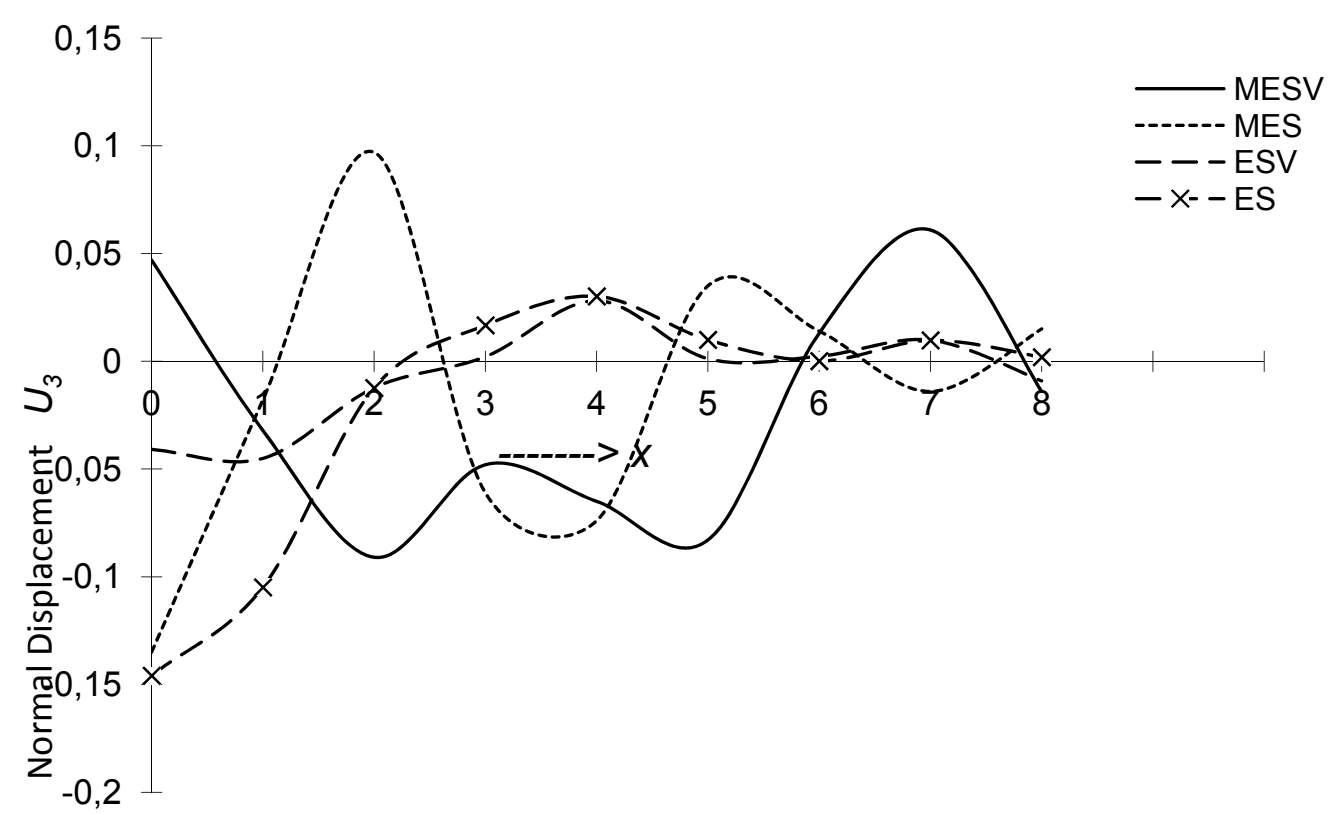

Fig.2. Variations of normal distance $U_{3}\left(=2 u_{3} / F_{0}\right)$ due to normal point source with distance $x$.

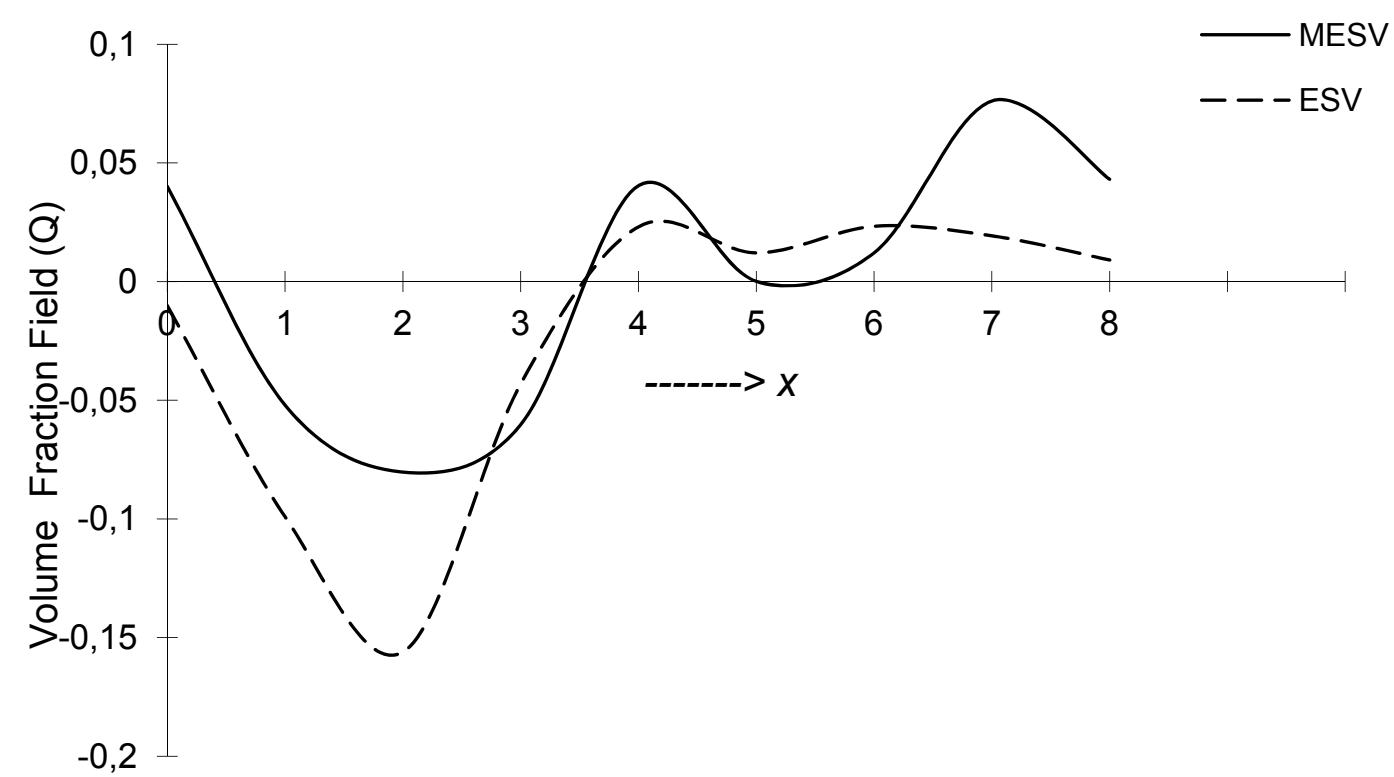

Fig.3. Variations of volume fraction field $Q\left(=2 q / F_{0}\right)$ due to normal point source with distance $x$. 
Figure 3 depicts the variations of volume fraction $Q$ with ' $x$ ' for MESV and ESV. The values of $Q$ decrease sharply in the range $0 \leq x \leq 2.5$ The values for MESV are greater than these for ESV in the initial range $0 \leq x \leq 2.5$ and then in the range $7 \leq x \leq 8$.

The variations of normal force stress $T_{33}$ with $r$ are shown in Fig.4. The values of $T_{33}$ start with a sharp decrease for MESV, MES and ES whereas for the case of ESV they start with a small increase. The magnitude of values of $T_{33}$ is largest for ES and smallest for MES.

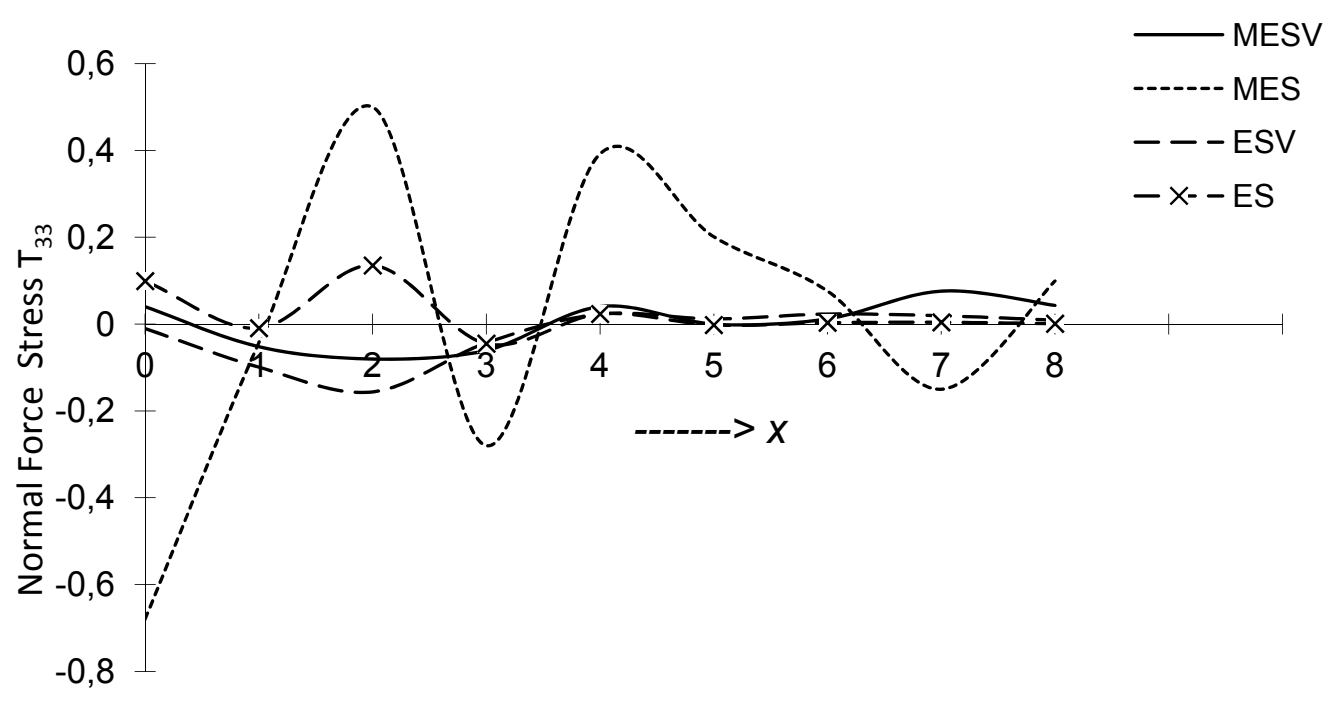

Fig.4. Variations of normal force stress $T_{33}\left(=2 t_{33} / F_{0}\right)$ due to normal point source with distance $x$.

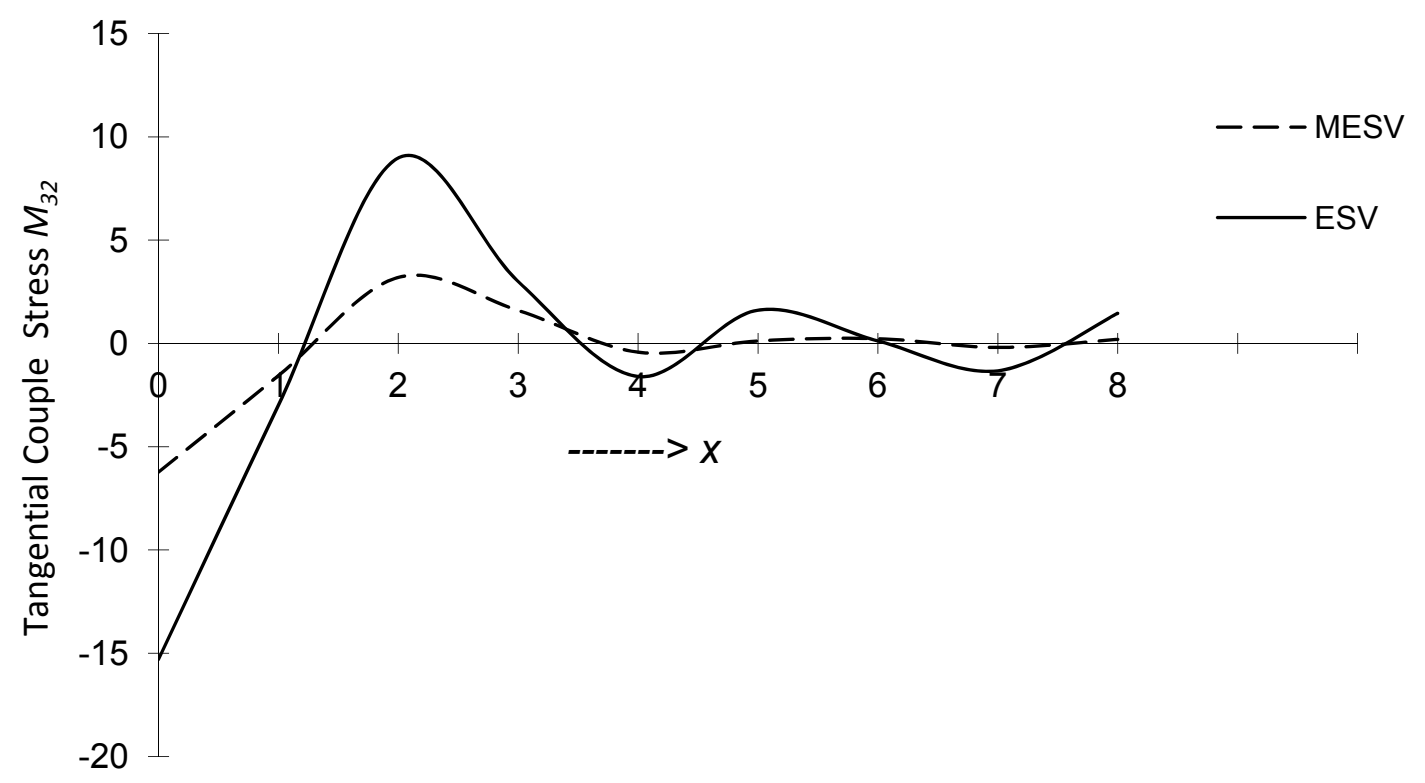

Fig.5. Variations of tangential couple stress $M_{32}\left(=2 m_{33} / F_{0}\right)$ due to normal point source with distance $x$. 
Figure 5 depicts the variations of $M_{32}$ with ' $x$ '. Beginning with a small decrease in the range $0 \leq x \leq 1.5$, the value of couple stress for MESV and MES start to grow up with a small variation. The behavior of variation of $M_{32}$ for both the cases is same whereas their corresponding values are different.

\section{Conclusion}

A significant effect of voids and micropolarity has been observed from the above numerical discussion. The magnitude of variations of normal displacement, normal force stress and tangential couple stress is observed for a mechanical normal point source. The void effect is appreciable in the mechanical sources. It is observed that the components of displacement, force stress, volume fraction field have large values which become smaller and smaller with the increase in the value of distance ' $x$ '.

\section{Nomenclature}

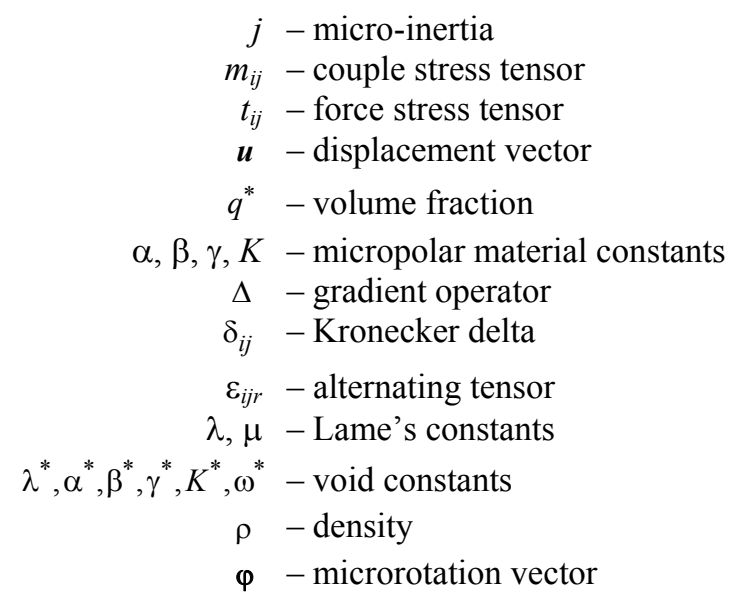

\section{References}

Chandrasekharaiah D.S. (1989): Complete solution in the theory of elastic materials with voids-II. - The Quarterly Journal of Mechanics and Applied Mathematics, vol.42, pp.41-54.

Cosserat E. and Cosserat F. (1909): Theories des Corp Formables. - Paris- Aherrman.

Cowin S.C. and Nunziato J.W. (1983): Linear elastic materials with voids. - Journal of Elasticity, vol.13, pp.125-147.

Eringen A.C. (1966): Linear theory of micropolar elasticity. - Journal of Mathematical and Mechanics, vol.15, pp.909924.

Eringen A.C. (1968): Theory of micropolar elasticity in fracture. - Academic Press, NewYork, vol.2, pp.621-729.

Eringen A.C. and Suhubi E.S. (1964): Non-linear theory of simple micropolar solids I. - International Journal of Engineering Sciences, vol.2, pp.189-203.

Honig G. and Hirdes V. (1984): A method for the numerical inversion of the Laplace transforms. - Journal of Computational and Applied Mathematics, vol.10, pp.113-132.

Iesan D.A. (1985): Shock waves in micropolar elastic materials with voids. - Analele Stiintifice ale Universitatu Al. 1. Cuza din lasi Seria Noua Sectiunea la matematica, vol.3, pp.177-186.

Kumar R. and Ailawalia P. (2007): Interaction in a micropolar thermoelastic medium with voids due to distributed loads; Int. J. Applied Mechanics and Engng., vol. 12, pp. 987-1007.

Kumar R., Deswal S. and Tomar S.K. (2002): A note on surface wave dispersion of a 1-layer micropolar liquid saturated half-space. - ISET Journal of Earthquake Technology, Technical note, vol.39, pp.367-382. 
Kumar R. and Kumar R. (2011): Wave propagation in transversely isotropic generalized thermoelastic half space with voids under initial stress. - Multidis. Modeling Materials Struct., vol.17.

Kumar R., Kumar Rajeev (2009): Analysis of waves motion in transversely isotropic medium with voids under a inviscid liquid layer. - Can. J. Phys., vol.87, pp.377- 388.

Madeo A. and Gavrilyuk S. (2010): Propagation of acoustic waves in porous media and their reflection and transmission at a pure-fluid/porous-medium permeable interface. - European J. Mech.-A/Solids, vol.29, No.5, pp.897-910.

Marin M. (1996a): On elastostatics of micropolar materials with voids. - Bull. Stiinca Univ. Politech. Ser. Math., vol.41, pp.29-37.

Marin M. (1996b): Generalized solutions in elasticity of micropolar bodies with voids. - Revista dele Academia Canaria de Ciencias, vol.8, pp.101-106.

Marin M. (1998): A temporally evolutionary equation in elasticity of micropolar bodies with voids. - Politehn. Univ. Bucharest Sci. Bull. Ser. Appl. Math. Phys., vol.60, pp.3-12.

Mindlin R.D. (1964): Microstructure in linear elasticity. - Archive for Rational Mechanics and Analysis, vol.16, pp. $51-78$.

Nunziato J.W. and Cowin S.C. (1979): A non-linear theory of elastic materials with voids. - Archive for Rational Mechanics and Analysis, vol.72, pp.175-201.

Scarpetta E. (1990): On the fundamental solution in micropolar elasticity with voids. - Acta Mechanica, vol.82, pp.151-158.

Scarpetta E. (1995): Wellposedness theorems for linear elastic materials with voids. - International Journal of Engineering Sciences, vol.33, pp.151-161.

Singh R. and Singh K. (2013): Eigen value approach in micropolar medium with voids. - Int. J. Applied Mechanics and Engng., vol.18, No.2, pp.521-536.

Tomar S.K. and Khurana A. (2011): Transmission of longitudinal wave through microporous elastic solid interface. Int. J. Engng. Sci. and Tech., vol.3, No.2, pp.12-21.

Wheeler L.T. and Isaak A.K. (1982): On voids of minimum stress concentration. - International Journal of Solids and Structures, vol.18, pp.85-89.

Received: October 27, 2013

Revised: May 3, 2014 